\title{
Relevance of Herniography for Accurate Diagnosis of Patent Processus Vaginalis in Cryptorchidism
}

\author{
R. Varela-Cives, A. Bautista-Casasnovas, P. Taboada-Santomil, E. Estevez-Martinez, \\ R. Mendez-Gallart, M. Pombo-Arias, R. Tojo-Sierra
}

Department of Pediatric Surgery and Pediatrics, Hospital Clinico Universitario, Universidad de Santiago de Compostela, Spain

\begin{abstract}
Objective: To clarify the role of peritoneography in assessing the patency of processus vaginalis (PV) in pediatric patients diagnosed with cryptorchidism.

Materials and Methods: We designed a prospective clinical trial to evaluate the patency of PV in boys presenting cryptorchidism. Herniography was performed in 310 prepubertal boys. Data about the morphology of PV was compared with operative findings in those surgically treated patients. Retractile and ectopic testes were excluded from the study. Results: Of the 376 undescended testes (310 patients), 281 cases were associated with an obliterated PV. Herniography revealed 95 cases of open PV in cryptorchid boys. The 244 normally descended testes had associated patent processus vaginalis in only 31 cases.

Conclusions: Herniography is the most relevant procedure for accurate diagnosis of persistent PV. The persistence of PV was significantly more frequent when the position of the testes is more cranial. The incidence of an open PV decreases with age.
\end{abstract}

Key words: testis; cryptorchidism; peritoneum; X-ray; inguinal hernia

Int Braz J Urol. 2008; 34: 57-62

\section{INTRODUCTION}

Cryptorchidism is one of the most frequent congenital anomalies in boys. Incidence ranges from 2 to $5 \%$ in full-term births reaching $30 \%$ in premature infants $(1,2)$. Data about the prevalence of persistent processus vaginalis in cryptorchidism is controversial. Reported prevalence varies from 2 to $98 \%$ depending on the author. The vast majority of published studies provide insufficient data to allow comparison (1-5).

In our study, we investigate the patency of PV in cryptorchidism. We carried out a blind, controlled, prospective clinical trial in which all patients underwent inguinal herniography (6). The present work focuses on patency of the processus vaginalis (PPV) in cryptorchidism and investigates relationships with age, position of the testis and type of cryptorchidism.

\section{MATERIALS AND METHODS}

We performed a blind, controlled, prospective clinical trial with pediatric patients diagnosed as having 
cryptorchidism. The study involved a total of 310 boys (376 testes), aged between 8 months and 11.5 years (mean 5.72 years) - 66 with bilateral cryptorchidism and 244 with unilateral cryptorchidism (153 on the right side, 91 on the left side). Only patients with no clinical evidence of hernia/hydrocele or other associated pathology were included in the study. None of the patients had previously undergone hormonal treatment or surgery in the inguinoscrotal region.

The patients were classified into three age groups: less than 2 years of age ( 40 boys with 48 undescended testes), $2-6$ years of age (132 boys with 158 undescended testes), and $6-12$ years of age (138 boys with 170 undescended testes).

The testis position was defined as nonpalpable, inguinal, external inguinal ring, scrotal entrance, high scrotal, or scrotal (normal), in view of the most caudal position obtainable by physical examination. Special care was taken to exclude patients with retractile or ectopic testes from the study. All patients were examined independently by the first two authors and any discrepancies were resolved by consensus.

Before treatment with human chorionic gonadotrophin, a peritoneogram was obtained in all patients using the procedure described by $\mathrm{Oh}$ et al. (7). Briefly, contrast material (dose in accordance with patient weight) was injected into the abdominal cavity via a $22 \mathrm{G}$ needle inserted along the midline at 2 to 3 $\mathrm{cm}$ below the umbilical fold. After $10 \mathrm{~min}$, when the inguinal peritoneal margin had been adequately delineated, anteroposterior radiography of the lower abdomen and upper thighs was obtained.

The peritoneogram is considered valid when the contrast medium delineates the peritoneal cavity medially and laterally to the inferior epigastric vessels, seen as a bilateral notch at the lower limit of the contrast line. The existence of a hernia or open PV can be ruled out when there is no evidence of fissures or irregularities in the peritoneum medial or lateral to the epigastric notch, and no evidence of contrast below the peritoneum (Figure-1). An open processus vaginalis is visible as evaginations of the peritoneum, beginning from lateral to inferior epigastric vessels at the level of the internal inguinal orifice and running obliquely and medially along the inguinal canal to the outer inguinal orifice, and then continuing in the caudalventral direction and in some cases extending into the scrotum (Figure-2). The processus vaginalis may be fully or only partially obliterated. If the open section is less than $2 \mathrm{~cm}$ long, the peritoneogram is considered normal.

Six complications $(0.51 \%)$ were noticed: one case of intestinal puncture resolved spontaneously, one contrast reaction needed corticoid therapy and there were four cases of peritoneal irritations. The peritoneogram obtained was valid in all cases.

This study was approved by the Ethical Committee of the Pediatrics Department of our hospital, and informed parental consent was obtained in all cases.

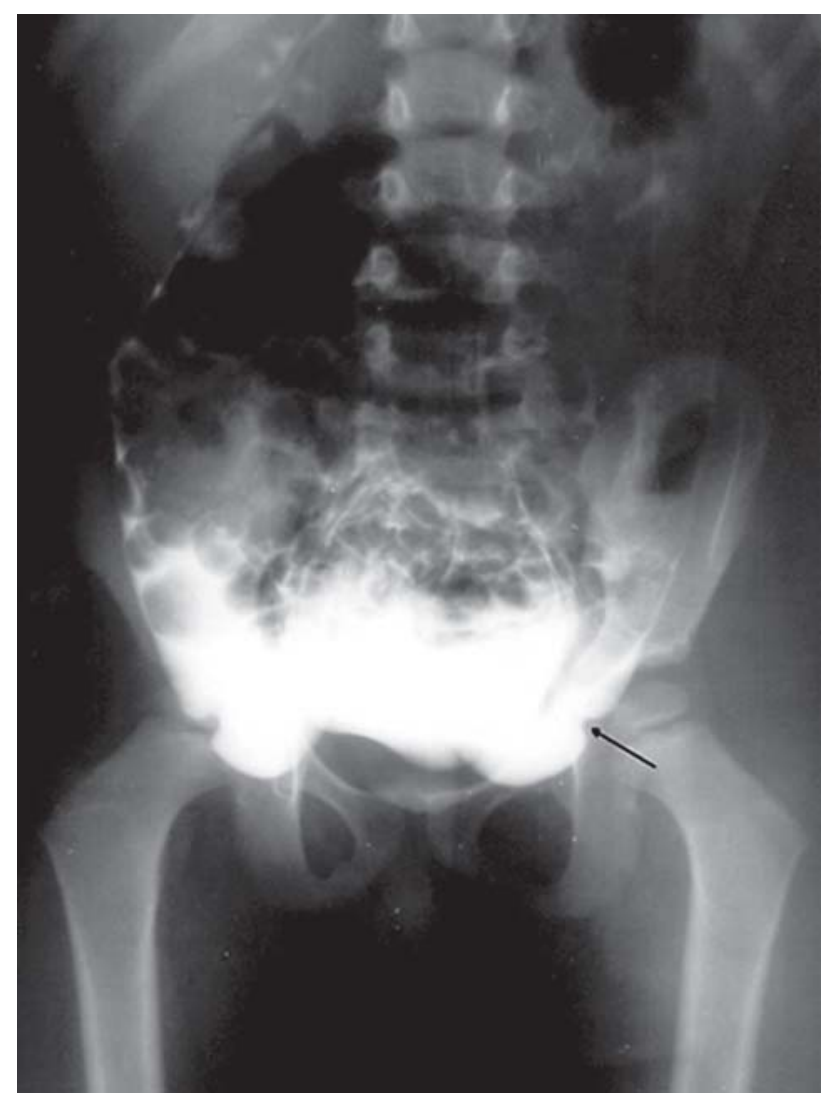

Figure 1 - Peritoneography. After intraperitoneal injection of contrast medium, anteroposterior pelvis $X$-ray shows no evidence of fissures or irregularities in the peritoneum medial or lateral to the epigastric notch (arrow), and no evidence of the presence of contrast medium below the peritoneum. 


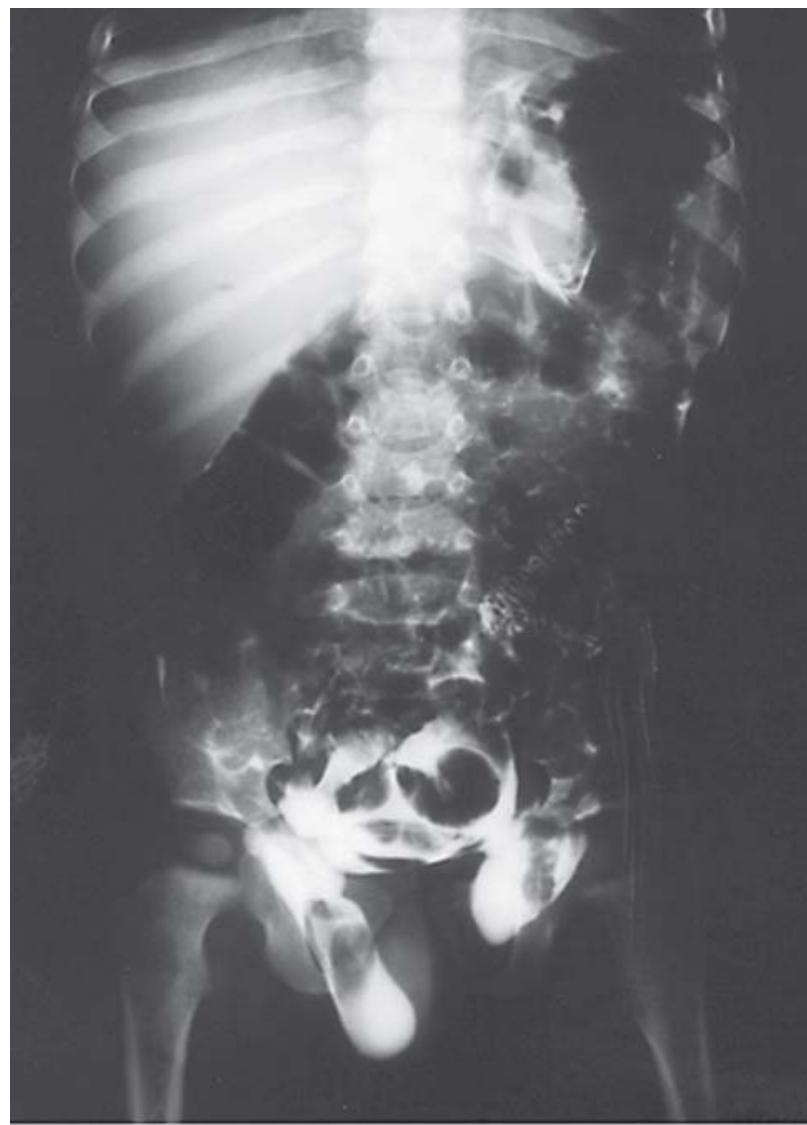

Figure 2 - Herniography of cryptorchid testis. After intraperitoneal injection of contrast medium, anteroposterior pelvis $X$-rays show an open peritoneal sac on the right side with descended testis and an open peritoneal sac on the left side with undescended testis.

Categorical variables were expressed as the percentage of occurrence with a confidence interval (CI) of $95 \%$ and continuous variables as the median. All statistical tests were two-tailed with $\mathrm{p}$ value <
0.05 denoting significance. For assessing baseline characteristics, categorical variables between groups were compared using the chi-squared test. The Statistical Package for Social Sciences (SPSS ${ }^{\circledR}$ 11.0) was employed for data analysis.

\section{RESULTS}

We detected 95 patent processus vaginalis (PPV) associated with the 376 undescended testes (25.3\%) and $31 \mathrm{PPV}$ associated with the 244 normally descended testes $(12.7 \%)$. This difference in prevalence was statistically significant $(\mathrm{p}=0.0001)$. Prevalence in the 0 - to 2-year age group was significantly higher $(\mathrm{p}<0.05)$ than in the 2 - to 6 -year age group. Prevalence in the $2-6$ year age group was also significantly higher that in the $6-12$ year age group $(\mathrm{p}<0.05)($ Table- 1$)$.

Morphological study indicated that the PPV associated with the undescended testes was open along their length and underdeveloped, never reaching the bottom of the scrotum.

PPV associated with undescended testes is shown in Table-2. Non-palpable testes and testes located in the inguinal region or external inguinal ring were associated with PPV in similar proportion (73/ 195, mean $37.4 \%)$. The percentage is significantly higher $(\mathrm{p}=0.0001)$ for testes located at the scrotal entrance $(19 / 117,16.2 \%)$, and for those located in high scrotal position $(3 / 64,4.7 \%$ with a p value 0.0230$)$. Analysis of the data following cross-classification by testis position and patient age indicates a significant prevalence of PPV between groups except for the

Table 1 - Patency of the processus vaginalis in descended and undescended testis related with age groups, $N$ of PPV / $N$ of testes (\%).

\begin{tabular}{lccl}
\hline Patients'Age & Undescended Testis & Descended Testis & p Value \\
\hline <2 years & $68.7 \%$ & $31.2 \%$ & $\mathrm{p}=0.001$ \\
2-6 years & $27.2 \%$ & $16 \%$ & $\mathrm{p}=0.0336$ \\
> 6years & $11.2 \%$ & $3.8 \%$ & $\mathrm{p}=0.0304$ \\
Total & $25.3 \%$ & $12.7 \%$ & $\mathrm{p}=0.0001$ \\
\hline
\end{tabular}

$P P V=$ patent processus vaginalis. 
Table 2 - Incidence of PPV related to testis location and age group. Values are shown in percentages. Number of undescended testes associated with PPV / Total number of undescended testes.

\begin{tabular}{|c|c|c|c|c|}
\hline \multirow[t]{2}{*}{ Testis Location } & \multicolumn{3}{|c|}{ Patients' Age (years) } & \multirow[b]{2}{*}{ Total } \\
\hline & $<2$ & $2-6$ & $>6$ & \\
\hline Nonpalpable & $50.0 \%$ & $40.0 \%$ & $35.75 \%$ & $41.23 \%$ \\
\hline Inguinal & $83.3 \%$ & $44.45 \%$ & $15.9 \%$ & $34.9 \%$ \\
\hline External inguinal ring & $81.8 \%$ & $46.6 \%$ & $8.7 \%$ & $36.7 \%$ \\
\hline Pubic & $63.6 \%$ & $18.25 \%$ & $3.9 \%$ & $16.2 \%$ \\
\hline High scrotal & - & - & $7.9 \%$ & $4.7 \%$ \\
\hline \multirow[t]{2}{*}{ Total } & $48 / 33$ & $158 / 43$ & $170 / 19$ & $376 / 95$ \\
\hline & $68.8 \%$ & $27.2 \%$ & $11.2 \%$ & $25.3 \%$ \\
\hline
\end{tabular}

$P P V=$ patent processus vaginalis.

non-palpable and high scrotal located testis. Considering differences in patient age and testis position (by chi-squared test of a three-dimensional table), we did not observe any statistically significant difference in the prevalence of PPV between unilaterally and bilaterally cryptorchidism, nor between right side and left side cryptorchidism.

\section{COMMENTS}

Previously published reports of the prevalence of PPV in cryptorchidism have been based on surgical findings. Only a few papers referred data obtained by diagnostic image procedures. Research articles, monographs and textbooks show a highly variable, surgically determined estimation of the prevalence of PPV ranging from 2 to $98 \%$. In some reports, prevalence is described with imprecise terms like "in most cases", "commonly", or "in practically all cryptorchid testes" (1-5). Data presented in these papers are based on previous authors' findings, and in a few cases are cited erroneously. It seems that existing reported data are simply repeated without critical review. Furthermore, most studies do not include sufficiently detailed patient data.

Nevertheless, valuable conclusions can be drawn from some studies. One controlled double-blind clinical trial reported a surgically determined PPV prevalence of $23 \%$ in a group of cryptorchid patients aged 5-12 years without clinical hernia or hydrocele or other associated pathology (8). A prospective study of hormonal therapy in cryptorchidism reported a surgical diagnosed PPV prevalence of $65 \%$ in a group of boys aged $1-8$ years (mean 4 years), but the authors do not clarify if patients with other (inguinal) pathologies were excluded from the study (9). The prospective study of Adamsen et al. considered a group of boys aged $4-9$ years (excluding those with clinical hernia and hydrocele), and found a surgically PPV prevalence of $77 \%$ (10). Another prospective clinical trial published in 1987 considered a group of patients aged $1-12$ years, again with exclusion of patients with hernia or other pathologies, and found a surgically determined PPV prevalence of $71 \%$. This study reported data on the width of the PPV, and suggested that the prevalence of PPV is related to the surgical location of the testis (11). In the previously reported papers, all of the patients had undergone previous hormone treatment. In a retrospective study, different authors reported a surgically determined PPV prevalence of $55 \%$ in a group of $2-19$ year old boys (mean 8.9 years) with acquired cryptorchidism (12). Another retrospective published study reported surgical findings of PPV in $23 \%$ of boys over 5 years of age with late-presenting acquired calescent versus $84 \%$ in children aged less than 5 years of age with early-presenting maldescent (overall prevalence 50\%) (13). Similar results were obtained by other authors who found a prevalence of surgically determined PPV 
of $28 \%$ in patients with acquired maldescent versus $100 \%$ in patients with early-presenting maldescent (14). Cendron et al. found that the prevalence of PPV during surgery was $87 \%$ for unilateral undescended testes versus $71 \%$ for bilateral undescended testes in cryptorchid boys aged $1-9$ years (15). Favorito et al. found a surgical prevalence of PPV of $57.6 \%$ in a group of 102 patients (137 testes) aged between 1 and 33 years of age (mean 10.3). There was no difference reported in the frequency of PPV between the age groups (16). A very interesting paper focusing on the role of PV in cryptorchidism reported a surgically determined PPV prevalence of $31 \%$ in a group of patients who had not responded to hormone treatment versus $56 \%$ in patients who had not received such treatment before surgery (17).

The disparity in percentages of PPV prevalence in previous published data may also be due to differences in sample characteristics, study design or in the surgeon's subjective assessment of intraoperative PV morphology.

Based on these studies, we feel that preoperative assessment of PPV is essential for validation of surgical findings. Patency of the vaginal process could be defined using Magnetic Resonance Imaging or ultrasound scan, but the most reliable nonsurgical method for the detection of PPV is positive contrast peritoneography (herniography). Peritoneography is a valid diagnostic procedure, which shows a sensitivity and specificity close to $100 \%$ without false positives and few false negatives (1821). Two studies showed an accurate diagnosis of PPV based on peritoneography findings in $94 \%$ of the patients $(22,23)$.

The incidence of PPV in undescended testes is twice that of the normal descended testes. In the undescended testis, the patency of the processus vaginalis is related to both age and testis position. In patients aged less than 2 years, PPV prevalence was about $70 \%$. This percentage dropped dramatically to $27.2 \%$ in patients aged $2-6$ years and to $11.2 \%$ in patients aged $6-12$ years. Undescended testes located cranial to the external inguinal ring were associated with PPV significantly more frequently than undescended testes located more caudally. There was no difference in the frequency of the PPV between unilateral or bilateral cryptorchidism, or between right or left side cryptorchidism.

The prevalence of surgically-determined PPV is evidently expected to be lower in patients undergoing primary surgery than in patients undergoing surgery after failure of hormone treatment, since hormone treatment cannot be effective when the processus vaginalis is patent $(6,10,11,17,22)$. We think that hormone therapy would be useful in avoiding surgery in a group of cryptorchid patients with PV obliterated. Our study showed that the prevalence of PPV demonstrated after orchidopexy in 186 patients who did not respond to hormone treatment was $41.0 \%$. In the same group of patients, prevalence of PPV diagnosed by peritoneography was $23.5 \%$. There was excellent concordance ( $\mathrm{kappa}=0.90,95 \%$ confidence interval $0.83-0.96, p<0.00005$ ) between the PV characteristics obtained by herniography and the surgical findings reported after orchidopexy (23).

We can conclude that the incidence of PPV in cryptorchid boys is clearly influenced by testicular location and patients' age.

\section{CONFLICT OF INTEREST}

None declared.

\section{REFERENCES}

1. Leung AK, Robson WL. Current status of cryptorchidism. Advances in Pediatrics. 2004; 51: 35177.

2. Rabinowitz R, Hulbert WC Jr: Cryptorchidism. Pediatr Rev. 1994; 15: 272-4.

3. Wolf CK, Maizels M, Furness PD 3rd: The undescended testicle. Compr Ther. 2001; 27: 11-7.

4. Rajfer J: Congenital Anomalies of the Testis. In: Walsh PC, Campbell's Urology. Philadelphia, W B Saunders, 5th ed., 1986; pp. 1947-68.

5. Rozanski TA, Bloom DA: The undescended testis. Theory and management. Urol Clin North Am. 1995; 22: 107-18.

6. Varela Cives R, Bautista Casasnovas A, Alonso Martin A, Pombo Arias M, Tojo Sierra R: The influence of patency of the vaginal process on the efficacy of 
hormonal treatment of cryptorchidism. Eur J Pediatr. 1996; 155: 932-6.

7. Oh KS, Dorst JP, White JJ, Haller JA Jr, Heller RM, James AE, et al.: Positive-contrast peritoneography and herniography. Radiology 1973; 108: 647-54.

8. Bertelsen A, Thorup J, Pedersen PV, Mauritzen K, Skakkebaek NE: Intranasal LH-RH treatment of cryptorchidism. A clinical trial and 5 years follow-up. Eur J Pediatr. 1987; 146 (Suppl 2): S40-1.

9. Giannopoulos MF, Vlachakis IG, Charissis GC: 13 Years' experience with the combined hormonal therapy of cryptorchidism. Horm Res. 2001; 55: 33-7.

10. Adamsen S, Aronson S, Borjesson B: Prospective evaluation of human chorionic gonadotropin in the treatment of cryptorchidism. Acta Chir Scand. 1989; 155: 509-14.

11. Hazebroek FW, de Muinck Keizer-Schrama SM, van Maarschalkerweerd M, Visser HK, Molenaar JC: Why luteinizing-hormone-releasing-hormone nasal spray will not replace orchiopexy in the treatment of boys with undescended testes. J Pediatr Surg. 1987; 22: 117782.

12. Meijer RW, Hack WW, van der Voort-Doedens LM, Haasnoot K, Bos SD: Surgical findings in acquired undescended testis. J Pediatr Surg. 2004; 39: 1242-4.

13. Davey RB: Undescended testes: early versus late maldescent. Pediatr Surg Int. 1997; 12: 165-7.

14. Donnell SC, Rickwood AM, Jee LD, Jackson M: Congenital testicular maldescent: significance of the complete hernial sac. Br J Urol. 1995; 75: 702-3.

15. Cendron M, Huff DS, Keating MA, Snyder HM 3rd, Duckett JW: Anatomical, morphological and volumetric analysis: a review of 759 cases of testicular maldescent. J Urol. 1993; 149: 570-3.

16. Favorito LA, Costa WS, Sampaio FJ: Relationship between the persistence of the processus vaginalis and age in patients with cryptorchidism. Int Braz $\mathbf{J}$ Urol. 2005; 31: 57-61.

17. Herzog B, Rosslein R, Hadziselimovic F: The role of the processus vaginalis in cryptorchidism. Does a patent processus vaginalis have a prognostic importance for predicting subsequent fertility? Eur J Pediatr. 1993; 152 (Suppl 2): S15-6.

18. Lawrenz K, Hollman AS, Carachi R, Cacciaguerra S: Ultrasound assessment of the contralateral groin in infants with unilateral inguinal hernia. Clin Radiol. 1994; 49: 546-8.

19. Jewett TC Jr, Kuhn IN, Allen JE: Herniography in children. J Pediatr Surg. 1976; 11: 451-4.

20. Iarchy J: Peritoneography, a safe method to assess the bilaterality of inguinal hernias in children with an obvious unilateral hernia or cryptorchidism (100 cases). Acta Chir Belg. 1983; 83: 253-60.

21. Tollefsen I, Johannessen F: How to avoid complications in herniography. Eur J Radiol. 1999; 31: $177-81$.

22. White JJ, Shaker IJ, O KS, Murphy J, Engel BS, Haller JA Jr: Herniography: a diagnostic refinement in the management of cryptorchidism. Am Surg. 1973; 39: 624-9.

23. Varela-Cives R, Bautista-Casasnovas A, Gude F, Cimadevila-Garcia A, Tojo R, Pombo M: The predictive value of inguinal herniography for the diagnosis and treatment of cryptorchidism. J Urol. 2000; 163: 964-7.

Accepted after revision:

September 20, 2007

\section{Correspondence address:}

Dr. R. Varela-Cives

Department of Pediatric Surgery

Hospital Clinico Universitario de Santiago

C/Choupana s/n. 15706

Santiago de Compostela, Spain

Fax: + 3498195-0518

E-mail:pdvarela@usc.es 